\title{
A Cyst in Connection with the Right Fallopian Tube, arising probably from an Accessory Fallopian Tube.
}

By R. Hamiton Beli, M.A., M.B. (Cantab.), M.R.C.P. (Lond.), Physician to Out-Patients, Samaritan Free Hospital; Obstetrical Tutor and Registrar, St. Thomas's Hospital.

Is the November number of the Journal of Obstetrics and Gynceology of the British Empire there was a paper by Mr. Handley "On the Origin from Accessory Fallopian Tubes of Cysts of the Broad Ligament Situated above the Tube." This paper was based on the examination of three specimens in the Museum of the Royal College of Surgeons, and one removed by Mr. Malcolm when operating for an ovarian cyst. It seemed to me to possess great pathological interest, particularly because it dealt with a problem which was occupying my attention at the time.

The tumour which is the subject of this short paper I believe to be an example of these cysts arising in accessory Fallopian tubes, and if this view be correct it adds a clinical to the pathological interest, from the fact that it caused symptoms leading the patient to consult a doctor, first in Italy, and subsequently in England, and ultimately to consent to abdominal section.

The patient, an Italian woman (C. A.), without any knowledge of English, was admitted to St. Thomas's Hospital under the care of Dr. Cullingworth, March, 1903. The specimen, which was removed by abdominal section March 26th, was handed to me for examination, and I am indebted to Dr. Cullingworth for permission to publish the result of my investigation.

The clinical history of the case was briefly as follows:Previous to this illness she had always been a healthy woman; had been married fifteen years, but never pregnant. Up to two years ago menstruation had been regular, but somewhat profuse. The first indication of any pelvic trouble was a change in the character of her periods, which recurred at much more frequent intervals, often twice a month, but with a scanty instead of a profuse flow. Concurrently with this she had pain in the right hypogastric and lumbar regions, and also severe headaches. Two years previous to admission she had been told by an Italian doctor that there was a small tumour in the right side. This had now enlarged so as to be a lump in the right 
iliac fossa appreciable to herself, and the pain had continued and become more severe. She therefore came into hospital prepared to undergo an operation. On palpation of the abdomen a smooth swelling could be felt on the right side, rising about two and a half inches above Poupart's ligament. It was cystic in nature, and appeared to be fixed. Per vaginam the swelling could be felt depressing the vaginal roof posteriorly and to the right. It was smooth, round, soft, elastic and fixed, and was continuous with the swelling felt in the abdomen. The uterus was of normal size, but it was pushed forwards and a little to the left, and its mobility was impaired.

At the operation, after adhesions had been separated, a large cystic mass was extruded from the wound. It consisted apparently of two portions-a hydrosalpinx of the right Fallopian tube, and a large darker cyst, bound to the hydrosalpinx by strong adhesions. The ovary was seen lying separately. The hydrosalpinx and adherent cyst were removed together. The ovary was left in situ.

The parts removed were handed over to me for examination. I endeavoured first to find out whether there was any communication between the hydrosalpinx and the large cyst. The latter was placed above the tube, and was adherent to it for rather more than an inch along its upper border. I passed a fine probe down the tube, which was very slightly dilated at its uterine end, but could not make it pass from the dilated ampulla into the large cyst. Failing in this, I carefully separated the adhesions until the two were connected by a thin pedicle only, less than an eighth of an inch in diameter. Further efforts were then made to pass the probe, but without success. I then snipped through the pedicle and separated the cyst, no drop of fluid escaping. Whilst separating adhesions another small cyst was discovered tucked in between the hydrosalpinx and the large cyst, but communicating with neither. The large cystic mass measured $3 \frac{1}{2}$ in. by $2 \frac{1}{2}$ in. by $2 \frac{3}{4}$ in. The wall was exceedingly thin at places and translucent, especially at its upper pole, away from the Fallopian tube. When opened the contents were seen to be a clear yellowish fluid. On opening the hydrosalpinx the fimbriæ could be seen radiating from a central band of fibrous tissue, which represented the abdominal ostium. The contents of the tube were a clear, thin, practically colourless fluid.

Various suggestions were made as to the origin of the large cyst, for example that it was a perimetric cystoma, but it seemed clear that microscopic examination of the wall was necessary before a definite opinion could be formed. The first sections cut not proving very 


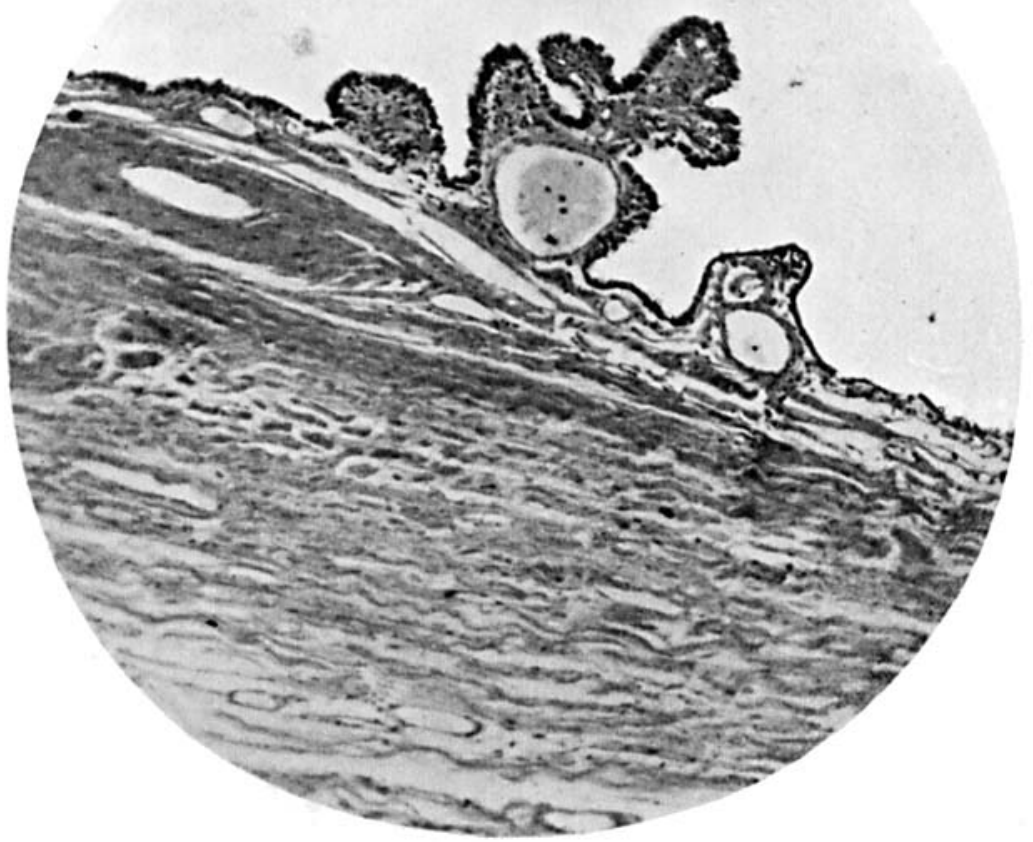

A. Wall of cyst under low power showing plicæ.

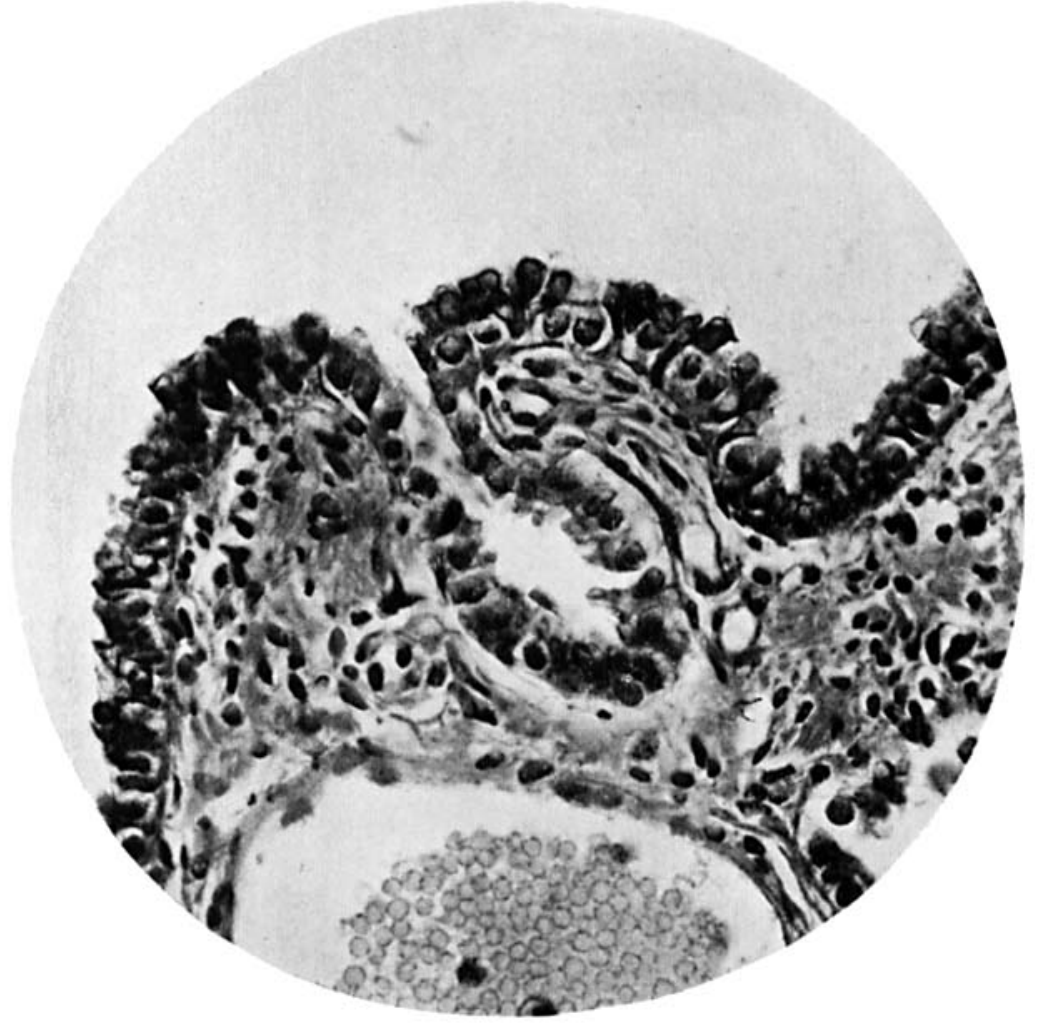

$B$. Portion of plica under high power, showing the lining of definite columnar epithelium. 
satisfactory, further portions of the wall were removed. On examining the interior of the cyst slightly raised strands were seen, and on the supposition that these might possibly be plicæ, the sections were made to include them. Sections were also cut horizontally through the wall, and stained by Van Giesen's method, to ensure that a small quantity of muscle which might be present should not be overlooked. The result of this section I may state at once. The fibrous tissue, stained red, very much predominated, but here and there were small areas stained yellow, with well-marked long nuclei, stained a dark yellowish brown, and there can, I think, be no doubt that these areas represented patches of muscular tissue, which had escaped the general fibrosis.

The perpendicular section was stained with hæm-alum and eosin. After several fruitless efforts a definite plica was discovered, and to prove that it was a ridge and not a fold serial sections were cut. The plica could be seen in every one of the series.

Detailed description of one section. The wall is seen to consist mainly of fibrous tissue. At one or two places are a few fibrils with long nuclei which appear to be muscle fibres, but this is not nearly so obvious as in the section stained by Van Giesen's method. No peritoneal lining can be made out on the outer surface of the cyst. (In regard to this I would point out that the wall was exceedingly thin and the sections consequently difficult to cut, so that it may be that the delicate peritoneum was lost in the preparation, or on the other hand that this large cyst growing above the Fallopian tube may have ruptured the peritoneum and entered the peritoneal cavity.)

The inner surface is lined throughout by epithelium, partly flattened, partly cubical, and in the neighbourhood of the plica, and over the plica itself, columnar in character. The plica is seen to consist of two or three folds of connective tissue, covered throughout with a columnar epithelium, and having at its base a large blood vessel. Close to the large plica is a smaller fold, with its epithelial covering more flattened, but with two distinct blood vessels at its base, an artery and a vein. Further down the section is a space lined on both sides by a columnar epithelium. This appears to me to be without much doubt a sub-plical space. Vide Figs. 1 and 2.

This perpendicular section, together with the horizontal one stained by Van Giesen's method, seems to afford a fairly certain proof of the origin of this cyst from an accessory Fallopian tube. The points to which I would specially direct attention are (1) the muscle tissue found in the wall, (2) the epithelial lining, definitely 
columnar in places, (3) and most convincing, the serial sections of a well-marked plica. If this view be correct the specimen is interesting as showing that cysts arising in this manner may reach such a size as to present identical clinical phenomena with a hydrosalpinx of the main Fallopian tube.

Sections were also made of the wall of the smaller cyst mentioned above, but they were still more difficult to cut, and were not very successful. Although I personally believe its origin to be similar to that of the large cyst, I am not able to bring forward definite proof of this, which must remain only a conjecture.

The sections were all cut and stained for me in the clinical laboratory of St. Thomas's Hospital, and I must express my debt to Mr. Dudgeon, the superintendent of the laboratory.

The woman made a rapid and uninterrupted recovery from the operation. 